\title{
Renal impairment is closely associated with plasma aldosterone concentration in patients with primary aldosteronism
}

\author{
Akiyuki Kawashima', Masakatsu Sone', Nobuya Inagaki', Yoshiyu Takeda ${ }^{2}$, Hiroshi Itoh ${ }^{3}$, Isao Kurihara ${ }^{3}$, \\ Hironobu Umakoshi ${ }^{4}$, Takamasa Ichijo5, Takuyuki Katabami ${ }^{6}$, Norio Wada7, Yoshihiro Ogawa ${ }^{8}$, \\ Junji Kawashima9 ${ }^{9}$ Megumi Fujita ${ }^{10}$, Shozo Miyauchi ${ }^{11}$, Shintaro Okamura ${ }^{12}$, Tomikazu Fukuoka ${ }^{13}$, \\ Toshihiko Yanase ${ }^{14}$, Shoichiro Izawa ${ }^{15}$, Yuichiro Yoshikawa ${ }^{16}$, Shigeatsu Hashimoto ${ }^{17}$, Masanobu Yamada ${ }^{18}$, \\ Tatsuya Kai ${ }^{19}$, Tomoko Suzuki ${ }^{20}$ and Mitsuhide Naruse ${ }^{4,21}$ on behalf of the JPAS and JRAS groups ${ }^{\dagger}$
}

${ }^{1}$ Department of Diabetes, Endocrinology and Nutrition, Kyoto University, Kyoto, Japan, ${ }^{2}$ Department of Internal Medicine, Graduate School of Medical Science, Kanazawa University, Kanazawa, Japan, ${ }^{3}$ Department of Endocrinology, Metabolism and Nephrology, Keio University School of Medicine, Tokyo, Japan, ${ }^{4}$ Department of Endocrinology and Metabolism, National Hospital Organization Kyoto Medical Center, Kyoto, Japan, ${ }^{5}$ Department of Endocrinology and Metabolism, Saiseikai Yokohamashi Tobu Hospital, Yokohama, Japan, ${ }^{6}$ Division of Metabolism and Endocrinology, Department of Internal Medicine, St. Marianna University School of Medicine, Yokohama City Seibu Hospital, Yokohama, Japan, ${ }^{7}$ Department of Diabetes and Endocrinology, Sapporo City General Hospital, Sapporo, Japan, ${ }^{8}$ Department of Medicine and Bioregulatory Science, Graduate School of Medical Science, Kyushu University, Fukuoka, Japan, ${ }^{9}$ Department of Metabolic Medicine, Faculty of Life Science, Kumamoto University, Kumamoto, Japan, ${ }^{10}$ Division of Nephrology and Endocrinology, Faculty of Medicine, The University of Tokyo, Tokyo, Japan, ${ }^{11}$ Department of Diabetes and Endocrinology, Ehime Prefectural Central Hospital, Matsuyama, Japan, ${ }^{12}$ Department of Endocrinology, Tenriyorozu Hospital, Tenri, Japan, ${ }^{13}$ Department of Internal Medicine, Matsuyama Red Cross Hospital, Matsuyama, Japan, ${ }^{14}$ Department of Endocrinology and Diabetes Mellitus, Faculty of Medicine, Fukuoka University, Fukuoka, Japan, ${ }^{15}$ Department of Endocrinology and Metabolism, Tottori University Hospital, Yonago, Japan, ${ }^{16}$ Department of Endocrinology and Diabetes Mellitus, Misato Kenwa Hospital, Misato, Japan, ${ }^{17}$ Division of Nephrology, Hypertension, Endocrinology, and Diabetology/Metabolism, Fukushima Medical University Hospital, Fukushima, Japan, ${ }^{18}$ Department of Medicine and Molecular Science, Gunma University Graduate School of Medicine, Maebashi, Japan, ${ }^{19}$ Department of Cardiology, Saiseikai Tondabayahsi Hospital, Tondabayashi, Japan, ${ }^{20}$ Department of Public Health, School of Medicine, International University of Health and Welfare, Narita, Japan, and ${ }^{21}$ Center of Endocrine Diseases, Takeda General Hospital, Kyoto, Japan

${ }^{\dagger}$ (Details of the JPAS and JRAS groups are presented as Supplementary data)

Correspondence should be addressed to M Sone Email sonemasa@kuhp.kyoto-u. ac.jp

\section{Abstract}

Objective: Several clinical studies have reported that renal impairments are sometimes observed in patients with primary aldosteronism (PA). We analyzed the prevalence of renal impairments in PA patients and identified parameters that increase the risk for them.

Design: This is a retrospective cross-sectional study. We assessed the PA database established by the multicenter Japan PA study (JPAS). Data were also collected from patients with essential hypertension (EHT).

Methods: We compared the prevalences of proteinuria and lowered estimated glomerular filtration rate (eGFR) between patients with PA and age, sex, blood pressure and duration of hypertension-matched patients with EHT. We also performed logistic regression analysis to identify parameters that increase the risk for these renal impairments. Results: Among 2366 PA patients, the prevalences of proteinuria and lowered eGFR were 10.3 and $11.6 \%$, respectively. The prevalence of proteinuria was significantly higher in PA patients than matched-EHT patients (16.8 vs 4.4\%), whereas there was no significant difference in the prevalence of lowered eGFR (17.2 vs $15.0 \%)$. The logistic regression analysis also showed that the plasma aldosterone concentration (PAC) significantly increases the risk of proteinuria and lowered eGFR, independent of other known risk factors.

Conclusion: Plasma aldosterone levels are closely associated with renal impairment in patients with PA. This is contrast to our earlier finding that the PAC was not itself linearly associated with cardiovascular events such as stroke or ischemic heart disease. The mechanism underlying the kidney damage in patients with PA may differ from that affecting the cardiovascular system. 


\section{Introduction}

\section{Background}

Primary aldosteronism (PA) is characterized by inappropriate autonomous aldosterone secretion, which results in secondary hypertension, sodium retention, increased potassium excretion and suppression of plasma renin (1). In addition to causing secondary hypertension, evidence suggests PA is also associated with a higher risk of cardiovascular events than is essential hypertension (EHT), independent of blood pressure $(2,3,4)$.

The contribution of excess aldosterone to renal impairment has been widely reported. For example, rats fed a high-salt diet along with chronically administrated aldosterone exhibit intrarenal vascular and glomerular sclerosis and proteinuria. Treatment with a mineralocorticoid blocker ameliorated these effects, irrespective of blood pressure $(5,6,7,8)$. From a clinical viewpoint, Reincke et al. reported that PA patients show higher serum creatinine levels and lower glomerular filtration rates (GFRs) than EHT patients. They also reported that the initial potassium concentration, plasma aldosterone concentration (PAC) and presence of hypokalemia were all independent predictors of a lower GFR (9). However, whether or not there is a difference in the GFR between patients with PA and those with EHT remains controversial. Catena (10) and Sechi (11) reported that the GFR is higher in patients with PA than in those with EHT. Ribstein et al. reported that the GFR does not differ between PA and EHT patients (12). Rossi et al. showed that 24-h urinary albumin excretion was significantly higher in PA patients than EHT patients (13). A more detailed analysis has not yet been performed due to the limited number of enrolled patients in those studies.

\section{Objectives}

Low GFR and proteinuria are reportedly independent factors in cardiovascular disease (14) and are a public health concern all over the world (15). In the present study, therefore, we examined the prevalence of renal impairment defined as the presence of proteinuria or an eGFR $<60 \mathrm{~mL} / \mathrm{min} / 1.73 \mathrm{~m}^{2}$ among patients with PA and determined the factors associated with the reduced eGFR and proteinuria indicative of renal impairment.

\section{Patients and methods}

\section{Study design}

This study was conducted as a part of the Japan Primary Aldosteronism Study (JPAS) and is a retrospective crosssectional analysis. The nationwide PA registry in Japan was established at 29 centers, including 15 university hospitals and 14 city hospitals.

\section{Setting and participants}

PA patients who were diagnosed and underwent adrenal venous sampling (AVS) between January 2006 and October 2017 were enrolled. Patients eligible to participate in the JPAS were men and women aged 20-90 years. Patients whom the investigators deemed unsuitable were excluded.

The patients' clinical characteristics, biochemical findings and results of confirmatory testing were collected electronically using a web registry system. The present study was conducted using a data set validated in February 2018.

The diagnosis of PA was made in accordance with guidelines from the Japan Endocrine Society and the Japan Society of Hypertension $(16,17)$. PA was diagnosed based on positive case detection of a ratio of the PAC (measured in $\mathrm{ng} / \mathrm{dL}$ ) to plasma renin activity (PRA, measured in $\mathrm{ng} / \mathrm{mL}$ per hour) $>20$ and at least one positive result from a confirmatory test, including the captoprilchallenge test, saline-infusion test, furosemide-upright test or oral salt-loading test. Diagnosis of the PA subtype was based on AVS with adrenocorticotropic hormone (cosyntropin) stimulation. Adrenal vein cannulation was defined as successful if the selectivity index was $>5$. The selectivity index was defined as the ratio of the cortisol concentration in the adrenal vein to that in the inferior vena cava. The unilateral subtype of PA was defined as a lateralization index $>4$. The lateralization index was calculated by dividing the aldosterone-to-cortisol ratio on the dominant side by that on the nondominant side (18). In the diagnosis of PA subtype, patients with suspected autonomous cortisol secretion defined as serum cortisol levels $\geq 1.8 \mu \mathrm{g} / \mathrm{dL}$ after a $1 \mathrm{mg}$ dexamethasone suppression test were excluded (19).

We referred to the medical history of the enrolled PA patients and those with a history of renal disease, including renal carcinoma, urinary stones, pyelonephritis, nephritis, nephrotic syndrome, polycystic kidney disease, nephrectomy and other urological disorder were excluded 
from this analysis, as those conditions could distort the results of renal biochemical testing. We also excluded patients who were taking a mineralocorticoid antagonist (MRA), angiotensin-converting enzyme inhibitor (ACEI), angiotensin II receptor blocker (ARB), diuretics or beta blocker at the time of diagnosis, as those drugs interfere with the results of PRA and PAC assays, thereby preventing accurate interpretation of the influence of aldosterone on renal impairment. The prevalences of renal impairment, proteinuria and lowered eGFR among PA patients were compared with those among EHT patients treated at the Kyoto Medical Center. Of the 274 patients with EHT treated between January 2006 and December 2013, 128 were compared after matching for age, sex, systolic blood pressure (sBP) and duration of hypertension. These parameters were matched in the range of \pm 1 year (age), $\pm 3 \mathrm{mmHg}(\mathrm{sBP})$ or \pm 1 year (duration of hypertension).

\section{Variables}

We collected data on age, sex, duration of hypertension, BMI, sBP, diastolic blood pressure (dBP) and blood chemistry, including basal levels of adrenocorticotropic hormone (ACTH) and cortisol, $\mathrm{Na}+, \mathrm{K}+, \mathrm{Cr}, \mathrm{BUN}$, eGFR, uric acid (UA), PAC, PRA, aldosterone-to-renin ratio (ARR), fasting blood sugar (FBS), HbA1c (NGSP (National Glycohemoglobin Standardization Program)), total cholesterol (TC), triglyceride (TG), high-density lipoprotein cholesterol (HDL-C), and low-density lipoprotein cholesterol (LDL-C, calculated using Friedewald equation).

Patients were considered to have diabetes mellitus (DM) or dyslipidemia (DL) after confirming a history of DM or DL in the dataset or if they were taking medication for DM or DL. Diagnoses of DM and DL were made at each institution according to those guidelines $(20,21)$. Hypokalemia was considered to be present if serum potassium was lower than $3.5 \mathrm{mEq} / \mathrm{L}$ or if a patient was taking a potassium supplement. Oral potassium was supplemented if hypokalemia was present.

\section{Study size}

The number of PA patients registered was 2814 .

\section{Analyses}

The clinical characteristics of PA patients with renal impairment were compared with those of PA patients without renal impairment. A diagnosis of renal impairment was made when PA patients had an eGFR
$<60 \mathrm{~mL} / \mathrm{min} / 1.73 \mathrm{~m}^{2}$ or proteinuria $(+,++,+++)$. The eGFR was calculated using the predictive equation defined by Japanese Society of Nephrology (15): 194×(serum creatinine $)^{-1.094} \times(\text { age })^{-0.287}$ for men and $194 \times($ serum creatinine $)^{-1.094} \times(\text { age })^{-0.287} \times 0.739$ for women. Overt proteinuria was defined as a result of,+++ or +++ on the dip stick test, while trace proteinuria was defined as a result of \pm on the dip stick test. In qualitative urine analyses, thresholds of + and ++ are unified in Japan and defined a $30 \mathrm{mg} / \mathrm{dL}$ and $100 \mathrm{mg} / \mathrm{dL}$, respectively. The thresholds of \pm and +++ are not unified, but most kits available in Japan define those thresholds a $15 \mathrm{mg} / \mathrm{dL}$ and $300 \mathrm{mg} / \mathrm{dL}$, respectively (15). As a diagnosis of renal impairment can be made based on the presence of proteinuria or lowered eGFR, we analyzed the effect of PA on proteinuria and eGFR separately, comparing patients with and without proteinuria or lowered eGFR.

We also investigated the clinical differences between patients with sustained eGFR and those with reduced eGFR defined as eGFR $<60 \mathrm{~mL} / \mathrm{min} / 1.73 \mathrm{~m}^{2}$. We then categorized patients with/without proteinuria into five groups:,,,- \pm+++ and +++ , after which we compared PACs among the five groups.

\section{Statistical methods}

We used JMP ${ }^{\circledR}$ ver. 13.2.1 developed by the SAS Institute Inc. and Stata $^{\circledR} /$ SE ver. 14 developed by LightStone ${ }^{\circledR}$. Results are presented as the median (interquartile range) and frequencies (positive/total observations) unless otherwise stated. The Wilcoxon signed-rank test was used for quantitative variables. Pearson's $\chi^{2}$ test was used for categorical variables. Cuzick's non-parametric test was used to assess trends across ordered groups. To account for multiple comparisons, we defined statistical significance as $P<0.01$ (22). Spearman's rank correlation coefficients for parameters associated with renal impairment, proteinuria and lowered eGFR in the univariate analysis were deemed statistically significant when the correlation coefficient satisfied $r>0.4$ and $P<0.01$. When we found that two or more parameters showed a statistically significant correlation, we selected the most representative parameter and brought it into a logistic regression analysis. The risk of an event is expressed as the odds ratio with the $99 \%$ confidence interval ( $99 \% \mathrm{CI})$.

\section{Assay methods}

At all centers, PAC was determined using a radioimmunoassay (SPAC-S Aldosterone kits, Fuji Rebio, 
Co., LTD, Tokyo, Japan). The reference range for PAC with patients in a supine position was $3.0-15.9 \mathrm{ng} / \mathrm{dL}$. PRA was measured using a radioimmunoassay or enzyme immunoassay (EIA). The reference range for PRA with patients in a supine position was $0.3-2.9 \mathrm{ng} / \mathrm{mL} / \mathrm{h}$ (PRA-FR RIA kits, Fuji Rebio, Co., Ltd, Tokyo, Japan) at 17 centers, 0.2-2.3 ng/mL/h (PRA EIA kits, Yamasa, Co., Ltd, Choshi, Japan) at 8 centers and $0.2-2.7 \mathrm{ng} / \mathrm{mL} / \mathrm{h}$ (PRA RIA kits, Yamasa, Co., Ltd) at 4 centers.

In Japan, nearly all university hospitals and large city hospitals have introduced automated analyzers for urinalysis. According to the annual report of the Japan Medical Association External Quality Control Program in 2016, there are three major companies providing these automated analyzers in Japan. EIKEN CHEMICAL Co., Ltd. have the largest market share in Japan (42.5\%), followed by ARKRAY, Inc. (29.6\%) and Siemens Healthcare K. K. (22.4\%). Regarding the,+++ and +++ proteinuria levels, the thresholds do not differ between these three companies. For trace proteinuria $( \pm)$, the threshold is $15 \mathrm{mg} / \mathrm{dL}$ with the analyzers from EIKEN CHEMICAL Co., Ltd. and ARKRAY Inc.; the analyzer from Siemens Healthcare cannot detect trace proteinuria.

\section{Ethics}

This study was conducted in accordance with the Declaration of Helsinki and the guidelines for clinical studies published by the Ministry of Health and Labor, Japan and was approved by the Ethics Committee of the National Hospital Organization Kyoto Medical Center as the project-leading center and by the institutional ethics committees of the participating centers. The present retrospective study received ethical approval for the use of the opt-out consent method according to the Ethics Guidelines for Medical Research for Humans in Japan.

\section{Results}

\section{Participants}

Of 2814 enrolled PA patients, we excluded 208 patients who had a history of renal or urological diseases, including nephritis, nephrotic syndrome or urinary stones. Also excluded were patients prescribed a MRA $(n=48)$, ACE-I $(n=14)$, ARB $(n=108)$, diuretics $(n=14)$ or beta blocker $(n=56)$. Data from the remaining 2366 PA patients were analyzed.

\section{Descriptive data}

Baseline characteristics are summarized in Table 1. The prevalences of renal impairment, overt proteinuria and lowered eGFR were 19.7\% (419/2127), 10.3\% (216/2104) and $11.6 \%$ (270/2325), respectively. The prevalence of trace and overt proteinuria was $25.7 \%(541 / 2104)$. Patients with proteinuria were categorized into five groups. The proportions of patients with overt and trace proteinuria were 10.3 (216/2104) and 15.4\% (325/2104), respectively. The proportion of patients with overt proteinuria at each severity level was as follows: +, 7.8\% (164/2104); ++, 1.5\% $(32 / 2104)$ and,$+++ 1.0 \%(20 / 2104)$ (Table 2).

\section{Main results}

Factors associated with renal impairment, proteinuria and lowered eGFR in patients with PA.

Table 1 Baseline characteristics of all PA participants in JPAS. Data are presented as median (interquartile range).

\begin{tabular}{l} 
Characteristic \\
\hline Age, year \\
Sex, male, \% \\
BMI, kg/m² \\
Duration of hypertension, year \\
Systolic blood pressure, mmHg \\
Diastolic blood pressure, mmHg \\
Creatinine, mg/dL \\
Blood urea nitrogen, mg/dL \\
EGFR, mL/min/1.73 m² \\
Lowered eGFR, \% \\
Overt proteinuria, \% \\
Trace and overt proteinuria, \% \\
Renal impairment, \% \\
Uric acid, mg/dL \\
Na+, mEqL \\
$\mathrm{K}^{+}, \mathrm{mEq} / \mathrm{L}$ \\
Hypokalemia, \% \\
Fasting blood sugar, mg/dL \\
HbA1c (NGSP), \% \\
Diabetes mellitus, \% \\
Total cholesterol, mg/dL \\
Triglyceride, mg/dL \\
HDL cholesterol, mg/dL \\
LDL cholesterol, mg/dL \\
Dyslipidemia, \% \\
PAC, ng/dL \\
PRA, ng/mL/h \\
ARR, ng/dL per ng/mL/h \\
Laterality, unilateral, \% \\
ACTH, pg/mL \\
Cortisol, $\mu$ gg/dL \\
\end{tabular}

\begin{tabular}{c}
\hline All subjects \\
\hline $53(45-62)$ \\
43.6 \\
$24.2(21.8-27.1)$ \\
$5(2-11)$ \\
$140(129-152)$ \\
$86(78-95)$ \\
$0.69(0.59-0.82)$ \\
$13.0(11.0-15.6)$ \\
$78.8(67.8-92.1)$ \\
11.6 \\
10.3 \\
25.7 \\
19.7 \\
$5.2(4.3-6.2)$ \\
$142(140-143)$ \\
$3.8(3.4-4.1)$ \\
37.7 \\
$98(91-108)$ \\
$5.6(5.3-6.0)$ \\
16.6 \\
$191(170-215)$ \\
$105(74-148)$ \\
$54(45-66)$ \\
$111(93-132)$ \\
27.1 \\
$17.4(12.3-27.7)$ \\
$0.3(0.2-0.5)$ \\
$53.5(32-105)$ \\
27.2 \\
$19.3(11.3-31.0)$ \\
$11.3(8.6-15.2)$ \\
\\
\hline
\end{tabular}

ACTH, adrenocorticotropic hormone; ARR, aldosterone-to-renin ratio; BMI, body mass index; eGFR, estimated glomerular filtration rate; $\mathrm{HbA1}$, hemoglobin A1c; HDL, high-density lipoprotein; LDL, low-density lipoprotein; PAC, plasma aldosterone concentration; PRA, plasma renin activity. 
Table 2 Distribution of patients with PA according to their proteinuria level.

\begin{tabular}{lcccc}
\hline Result of proteinuria & & Number of patients & & Proportion, \% \\
\cline { 1 - 1 }+ & 1563 & 325 & 74.3 \\
+ & 164 & 15.4 \\
++ & 32 & 7.8 \\
+++ & 20 & 1.5 \\
\hline
\end{tabular}

In a univariate logistic regression analysis, we found that age, sex (male), BMI, duration of hypertension, sBP, UA, Na, K, hypokalemia, FBS, HbA1c, DM, TG, DL, PAC, ARR and laterality (unilateral) all significantly associated with renal impairment (Table 3 ). We next analyzed the factors associated with overt proteinuria, which is a component of renal impairment. In a univariate logistic regression analysis, age, sex (male), BMI, duration of hypertension, sBP, dBP, UA, Na, K, hypokalemia, FBS, HbA1c, DM, HDL-C, DL, PAC, ARR and laterality (unilateral) all significantly associated with proteinuria (Table 4). We also searched for factors associated with lowered eGFR, defined as eGFR $<60 \mathrm{~mL} / \mathrm{min} / 1.73 \mathrm{~m}^{2}$. In a univariate logistic regression analysis, age, duration of hypertension, UA, Na, hypokalemia, DM, TG, DL, PAC and ARR all significantly associated with lowered eGFR (Table 5).

Given that factors such as age, sex, duration of hypertension and prevalence of DM significantly associated with each of these renal impairments, we conducted a multivariate logistic regression analysis to clarify the association between renal impairments and PA-specific factors. Before carrying out that analysis, we checked the correlation between all pairs of these parameters using Spearman's rank correlation test

Table 3 Differences in clinical characteristics between PA patients with and without renal impairment. Data are presented as median (interquartile range). Renal-associated parameters including creatinine, blood urea nitrogen and eGFR were excluded from univariate logistic regression analysis as they are apparently associated with renal impairment.

\begin{tabular}{l}
\hline \\
Characteristic \\
\hline Age, years \\
Sex, male, \% \\
BMI, kg/m² \\
Duration of hypertension, years \\
Systolic blood pressure, mmHg \\
Diastolic blood pressure, mmHg \\
Creatinine, mg/dL \\
Blood urea nitrogen, mg/dL \\
EGFR, mL/min/1.73 m² \\
Trace and overt proteinuria, \% \\
Uric acid, mg/dL \\
Na, mEq/L \\
K, mEq/L \\
Hypokalemia, \% \\
Fasting blood sugar, mg/dL \\
HbA1c (NGSP), \% \\
Diabetes mellitus, \% \\
Total cholesterol, mg/dL \\
Triglyceride, mg/dL \\
HDL cholesterol, mg/dL \\
LDL cholesterol, mg/dL \\
Dyslipidemia, \% \\
PAC, ng/dL \\
PRA, ng/mL/h \\
ARR, ng/dL per ng/mL/h \\
Laterality, unilateral, \% \\
ACTH, pg/mL \\
Cortisol, $\mu$ gg/dL \\
\hline
\end{tabular}

\begin{tabular}{c}
\hline $\begin{array}{c}\text { Patients with renal } \\
\text { impairment }\end{array}$ \\
\hline$n=419$ \\
\hline $60(51-66)$ \\
$53.5 \%$ \\
$24.8(22.1-28.0)$ \\
$10(3-19.5)$ \\
$142(130-155)$ \\
$86(77-96)$ \\
$0.88(0.76-1.10)$ \\
$15.4(12.8-18.2)$ \\
$57.1(50.9-69.5)$ \\
65.7 \\
$5.8(4.8-6.8)$ \\
$142(141-144)$ \\
$3.7(3.2-4.0)$ \\
$51.0 \%$ \\
$102(92-117)$ \\
$5.8(5.4-6.4)$ \\
$25.7 \%$ \\
$190(169-217)$ \\
$120(89-163)$ \\
$50(42-63)$ \\
$111(91-135)$ \\
$36.1 \%$ \\
$21.5(14.0-37.9)$ \\
$0.3(0.2-0.5)$ \\
$66(37-139)$ \\
$38.0 \%$ \\
$21.0(13.0-34.6)$ \\
$11.8(9.2-16.0)$ \\
\\
\end{tabular}

\begin{tabular}{|c|}
\hline $\begin{array}{l}\text { Patients without } \\
\text { renal impairment }\end{array}$ \\
\hline$n=1708$ \\
\hline $52(44-61)$ \\
\hline $42.0 \%$ \\
\hline $24.0(21.7-26.8)$ \\
\hline $5(1-10)$ \\
\hline $140(128-152)$ \\
\hline $87(78-95)$ \\
\hline $0.66(0.57-0.77)$ \\
\hline $12.7(10.9-15.0)$ \\
\hline 81.9 (72.0-93.6) \\
\hline 16.5 \\
\hline $5.1(4.2-5.9)$ \\
\hline $142(140-143)$ \\
\hline $3.8(3.5-4.1)$ \\
\hline $34.9 \%$ \\
\hline $97(90-107)$ \\
\hline $5.6(5.3-6.0)$ \\
\hline $15.4 \%$ \\
\hline $191(170-214)$ \\
\hline $103(73-146)$ \\
\hline $54(45-66)$ \\
\hline $112(93-132)$ \\
\hline $25.5 \%$ \\
\hline $16.8(12.1-25.7)$ \\
\hline $0.3(0.2-0.5)$ \\
\hline $52(31-100)$ \\
\hline $25.5 \%$ \\
\hline $19.0(11.0-30.2)$ \\
\hline $11.1(8.4-14.9)$ \\
\hline
\end{tabular}

\begin{tabular}{|c|c|}
\hline $\begin{array}{c}\text { Mean } \\
\text { difference }\end{array}$ & Unadjusted $O R$ \\
\hline 6.29 & 1.06 \\
\hline- & 1.59 \\
\hline 0.89 & 1.05 \\
\hline 4.23 & 1.05 \\
\hline 3.47 & 1.01 \\
\hline 0.39 & 1.00 \\
\hline 0.27 & - \\
\hline 2.93 & - \\
\hline 22.8 & - \\
\hline- & - \\
\hline 0.71 & 1.44 \\
\hline 0.52 & 1.11 \\
\hline 0.14 & 0.61 \\
\hline- & 1.94 \\
\hline 6.12 & 1.01 \\
\hline 0.19 & 1.19 \\
\hline- & 1.90 \\
\hline 0.06 & 0.99 \\
\hline 13.0 & 1.002 \\
\hline 2.44 & 0.99 \\
\hline 0.46 & 1.00 \\
\hline- & 1.65 \\
\hline 8.12 & 1.02 \\
\hline 0.04 & 1.27 \\
\hline 38.6 & 1.002 \\
\hline- & 1.79 \\
\hline 3.32 & 1.01 \\
\hline 0.71 & 1.01 \\
\hline
\end{tabular}

\begin{tabular}{|c|c|}
\hline $99 \% \mathrm{Cl}$ & $\boldsymbol{P}$ \\
\hline $1.04-1.07$ & $<0.001$ \\
\hline $1.20-2.11$ & $<0.001$ \\
\hline $1.02-1.09$ & $<0.001$ \\
\hline $1.03-1.07$ & $<0.001$ \\
\hline $1.00-1.02$ & $<0.001$ \\
\hline 0.99-1.01 & 0.577 \\
\hline- & - \\
\hline- & - \\
\hline- & - \\
\hline- & - \\
\hline $1.29-1.60$ & $<0.001$ \\
\hline $1.04-1.19$ & $<0.001$ \\
\hline $0.46-0.79$ & $<0.001$ \\
\hline $1.46-2.58$ & $<0.001$ \\
\hline $1.00-1.01$ & 0.002 \\
\hline $1.02-1.37$ & 0.002 \\
\hline $1.35-2.65$ & $<0.001$ \\
\hline 0.99-1.00 & 0.977 \\
\hline $1.000-1.004$ & 0.002 \\
\hline 0.98-1.00 & 0.012 \\
\hline 0.99-1.01 & 0.810 \\
\hline $1.22-2.22$ & $<0.001$ \\
\hline $1.01-1.03$ & $<0.001$ \\
\hline $0.90-1.77$ & 0.061 \\
\hline $1.001-1.003$ & $<0.001$ \\
\hline $1.25-2.55$ & $<0.001$ \\
\hline $0.99-1.02$ & 0.068 \\
\hline 0.98-1.06 & 0.124 \\
\hline
\end{tabular}

ACTH, adrenocorticotropic hormone; ARR, aldosterone-to-renin ratio; BMI, body mass index; Cl, confidence interval; eGFR, estimated glomerular filtration rate; $\mathrm{HbA1c}$, Hemoglobin A1c; HDL, high-density lipoprotein; LDL, low-density lipoprotein; OR, odds ratio; PAC, plasma aldosterone concentration; PRA, plasma renin activity. 
Table 4 Differences in clinical characteristics between PA patients with and without proteinuria. Data are presented as median (interquartile range). Renal-associated parameters including creatinine, blood urea nitrogen, eGFR and proteinuria were excluded from univariate logistic regression analysis since they are apparently associated with renal impairment.

\begin{tabular}{l} 
Characteristic \\
\hline Age, years \\
Sex, male, \% \\
BMI, kg/m² \\
Duration of hypertension, years \\
Systolic blood pressure, mmHg \\
Diastolic blood pressure, mmHg \\
Creatinine, mg/dL \\
Blood urea nitrogen, mg/dL \\
EGFR, mL/min/1.73 m² \\
Lowered eGFR, \% \\
Uric acid, mg/dL \\
Na, mEq/L \\
K, mEq/L \\
Hypokalemia, \% \\
Fasting blood sugar, mg/dL \\
HbA1c (NGSP), \% \\
Diabetes mellitus, \% \\
Total cholesterol, mg/dL \\
Triglyceride, mg/dL \\
HDL cholesterol, mg/dL \\
LDL cholesterol, mg/dL \\
Dyslipidemia, \% \\
PAC, ng/dL \\
PRA, ng/mL/h \\
ARR, ng/dL per ng/mL/h \\
Laterality, unilateral, \% \\
ACTH, pg/mL \\
Cortisol, $\mu$ g/dL \\
\hline
\end{tabular}

\begin{tabular}{c}
\hline $\begin{array}{c}\text { Patients with } \\
\text { proteinuria }\end{array}$ \\
\hline$n=216$ \\
\hline $56(46-64)$ \\
$66.2 \%$ \\
$25.6(22.9-29.0)$ \\
$10(3.8-20)$ \\
$146(133-158)$ \\
$89(78-99)$ \\
$0.81(0.66-1.03)$ \\
$14.3(12.0-17.0)$ \\
$68.9(55.9-87.3)$ \\
31.2 \\
$5.6(4.7-6.8)$ \\
$143(141-144)$ \\
$3.6(3.0-3.8)$ \\
$62.3 \%$ \\
$103(93-127)$ \\
$5.9(5.3-6.7)$ \\
$34.9 \%$ \\
$187(166-209)$ \\
$122(90-163)$ \\
$49(41-58)$ \\
$110(92-135)$ \\
$37.2 \%$ \\
$26.4(16.4-40.9)$ \\
$0.4(0.2-0.5)$ \\
$73.3(39.5-161)$ \\
$47.9 \%$ \\
$20.0(12.9-33.5)$ \\
$11.7(8.7-15.7)$ \\
\hline \\
\hline
\end{tabular}

\begin{tabular}{|c|c|c|}
\hline $\begin{array}{c}\text { Patients without } \\
\text { proteinuria }\end{array}$ & Mean & \\
\hline$n=1888$ & difference & Unadjusted OR \\
\hline $53(45-62)$ & 2.40 & 1.02 \\
\hline $41.7 \%$ & - & 2.73 \\
\hline $24.0(21.7-26.8)$ & 1.69 & 1.09 \\
\hline $5(1.5-11)$ & 4.07 & 1.04 \\
\hline $140(128-152)$ & 5.63 & 1.02 \\
\hline 86 (78-95) & 2.44 & 1.01 \\
\hline $0.68(0.58-0.80)$ & 0.19 & - \\
\hline $13.0(11.0-15.1)$ & 1.65 & - \\
\hline 79.5 (68.9-92.4) & 10.2 & - \\
\hline 9.4 & - & - \\
\hline $5.1(4.3-6.1)$ & 0.54 & 1.30 \\
\hline $142(140-143)$ & 0.46 & 1.10 \\
\hline $3.8(3.5-4.1)$ & 0.30 & 0.35 \\
\hline $35.2 \%$ & - & 3.04 \\
\hline $98(90-107)$ & 12.1 & 1.01 \\
\hline $5.6(5.4-6.0)$ & 0.36 & 1.33 \\
\hline $15.5 \%$ & - & 2.92 \\
\hline $192(170-215)$ & 4.94 & 0.99 \\
\hline $104(74-48)$ & 10.8 & 1.00 \\
\hline $54(45-66)$ & 5.57 & 0.98 \\
\hline $112(93-132)$ & 0.32 & 0.99 \\
\hline $26.4 \%$ & - & 1.66 \\
\hline $17.1(12.1-26.3)$ & 11.1 & 1.02 \\
\hline $0.3(0.2-0.5)$ & 0.06 & 1.40 \\
\hline $52.5(31.6-101)$ & 40.1 & 1.001 \\
\hline $25.8 \%$ & - & 2.64 \\
\hline $19.6(11.3-31.0)$ & 1.81 & 1.00 \\
\hline $11.2(8.5-15.2)$ & 0.24 & 1.01 \\
\hline
\end{tabular}

\begin{tabular}{c}
\hline $\mathbf{9 9 \%} \mathbf{~ I ~}$ \\
\hline $1.00-1.04$ \\
$1.85-4.04$ \\
$1.05-1.14$ \\
$1.03-1.06$ \\
$1.01-1.03$ \\
$1.00-1.03$ \\
- \\
- \\
- \\
- \\
$1.14-1.49$ \\
$1.01-1.20$ \\
$0.25-0.49$ \\
$2.07-4.46$ \\
$1.01-1.02$ \\
$1.11-1.57$ \\
$1.95-4.36$ \\
$0.99-1.00$ \\
$0.99-1.00$ \\
$0.96-0.99$ \\
$0.99-1.01$ \\
$1.12-2.44$ \\
$1.01-1.03$ \\
$0.94-2.09$ \\
$1.001-1.002$ \\
$1.67-4.18$ \\
$0.99-1.01$ \\
$0.96-1.05$ \\
\end{tabular}

\begin{tabular}{c}
\hline \\
$P$ \\
\hline 0.003 \\
$<0.001$ \\
$<0.001$ \\
$<0.001$ \\
$<0.001$ \\
0.009 \\
- \\
- \\
- \\
- \\
$<0.001$ \\
0.004 \\
$<0.001$ \\
$<0.001$ \\
$<0.001$ \\
$<0.001$ \\
$<0.001$ \\
0.076 \\
0.051 \\
$<0.001$ \\
0.903 \\
0.001 \\
$<0.001$ \\
0.028 \\
$<0.001$ \\
$<0.001$ \\
0.439 \\
0.701 \\
\hline \\
\hline
\end{tabular}

ACTH, adrenocorticotropic hormone; ARR, aldosterone-to-renin ratio; BMI, body mass index; $\mathrm{Cl}$, confidence interval; eGFR, estimated glomerular filtration rate; HbA1c, hemoglobin A1 c; HDL, high-density lipoprotein; LDL, low-density lipoprotein; OR, odds ratio; PAC, plasma aldosterone concentration; PRA, plasma renin activity.

in order to avoid multicollinearity. As described earlier, we selected the most representative parameters when statistically significant correlation was observed between two or more parameters. We also excluded renal-associated parameters including $\mathrm{Cr}$, BUN, eGFR, proteinuria and lowered eGFR, since they are apparently associated with renal impairment. As a result, parameters described separately in Tables 6, 7 and 8 were selected and brought into the logistic regression analysis. The logistic regression analysis showed that PAC was associated with the presence of renal impairment, proteinuria and lowered eGFR, independent of other parameters (Tables 6, 7 and 8). We also found that the presence of renal impairment and proteinuria was significantly associated with the presence of hypokalemia (Tables 6 and 7).

\section{Other analyses}

Comparison of PACs according to the severity of proteinuria

When PA patients were divided into five groups according to the severity of their proteinuria (negative for proteinuria $(-), \pm,+,++,+++)$, Cuzick's non-parametric test for trend showed that as PAC was significantly increased so was the severity of proteinuria $(P<0.001)$ (Fig. 1).

\section{Comparison between patients with PA and those with EHT}

We compared the prevalences of renal impairment, proteinuria and lowered eGFR in PA patients in the JPAS with those in EHT patients at the Kyoto Medical Center (Table 9). The prevalences of renal impairment and lowered eGFR did not statistically differ between patients 
Table 5 Differences in clinical characteristics between PA patients with and without lowered eGFR. Data are presented as median (interquartile range). Renal-associated parameters including creatinine, blood urea nitrogen, eGFR and lowered eGFR were excluded from univariate logistic regression analysis since they are apparently associated with renal impairment.

\begin{tabular}{l} 
Characteristic \\
\hline Age, years \\
Sex, male, \% \\
BMI, kg/m² \\
Duration of hypertension, years \\
Systolic blood pressure, mmHg \\
Diastolic blood pressure, mmHg \\
Creatinine, mg/dL \\
Blood urea nitrogen, mg/dL \\
EGFR, mL/min/1.73 m² \\
Overt proteinuria, \% \\
Trace and overt proteinuria, \% \\
Uric acid, mg/dL \\
Na, mEq/L \\
K, mEq/L \\
Hypokalemia, \% \\
Fasting blood sugar, mg/dL \\
HbA1c (NGSP), \% \\
Diabetes mellitus, \% \\
Total cholesterol, mg/dL \\
Triglyceride, mg/dL \\
HDL cholesterol, mg/dL \\
LDL cholesterol, mg/dL \\
Dyslipidemia, \% \\
PAC, ng/dL \\
PRA, ng/mL/h \\
ARR, ng/dL per ng/mL/h \\
Laterality, unilateral, \% \\
ACTH, pg/mL \\
Cortisol, $\mu$ gg/dL \\
\hline
\end{tabular}

\begin{tabular}{|c|}
\hline $\begin{array}{l}\text { Patients with } \\
\text { lowered eGFR }\end{array}$ \\
\hline$n=270$ \\
\hline $62(55-67)$ \\
\hline $49.3 \%$ \\
\hline $24.7(22.0-27.6)$ \\
\hline $10(3-20)$ \\
\hline $142(130-155)$ \\
\hline $84(76-94)$ \\
\hline $1.02(0.82-1.19)$ \\
\hline $16.6(14.1-20.0)$ \\
\hline $53.0(46.9-56.8)$ \\
\hline 27.4 \\
\hline 44.9 \\
\hline $6.1(5.0-7.0)$ \\
\hline $142(141-144)$ \\
\hline $3.7(3.3-4.1)$ \\
\hline $46.7 \%$ \\
\hline 102 (92-115) \\
\hline $5.8(5.5-6.3)$ \\
\hline $22.4 \%$ \\
\hline 194 (170-218) \\
\hline 115 (87-163) \\
\hline $51(43-65)$ \\
\hline $111(91-136)$ \\
\hline $37.7 \%$ \\
\hline $20.3(13.3-34.8)$ \\
\hline $0.3(0.2-0.5)$ \\
\hline 65 (37-130) \\
\hline $31.3 \%$ \\
\hline $21.6(13.0-37.3)$ \\
\hline $12.0(9.5-16.3)$ \\
\hline
\end{tabular}

\begin{tabular}{c}
\hline $\begin{array}{c}\text { Patients without } \\
\text { lowered eGFR }\end{array}$ \\
\hline$n=2055$ \\
\hline $52(44-61)$ \\
$42.9 \%$ \\
$24.1(21.7-27.0)$ \\
$5(1.5-10)$ \\
$140(129-152)$ \\
$87(78-95)$ \\
$0.67(0.57-0.77)$ \\
$12.9(11.0-15.0)$ \\
$81.8(71.8-93.8)$ \\
8.0 \\
23.2 \\
$5.1(4.2-6.0)$ \\
$142(140-143)$ \\
$3.8(3.4-4.0)$ \\
$36.5 \%$ \\
$97(90-107)$ \\
$5.6(5.3-6.0)$ \\
$15.9 \%$ \\
$191(170-214)$ \\
$102(74-146)$ \\
$54(45-66)$ \\
$111(93-131)$ \\
$25.9 \%$ \\
$17.0(12.2-26.9)$ \\
$0.3(0.2-0.5)$ \\
$53(32-102)$ \\
$26.7 \%$ \\
$19.0(11.0-30.1)$ \\
$11.1(8.4-15.0)$ \\
\\
\end{tabular}

\begin{tabular}{|c|c|}
\hline $\begin{array}{c}\text { Mean } \\
\text { difference }\end{array}$ & Unadjusted OR \\
\hline 9.34 & 1.09 \\
\hline- & 1.29 \\
\hline 0.24 & 1.01 \\
\hline 5.35 & 1.06 \\
\hline 2.75 & 1.01 \\
\hline 1.44 & 0.99 \\
\hline 0.39 & - \\
\hline 4.18 & - \\
\hline 33.9 & - \\
\hline- & - \\
\hline- & - \\
\hline 0.88 & 1.53 \\
\hline 0.46 & 1.10 \\
\hline 0.01 & 0.95 \\
\hline- & 1.53 \\
\hline 3.8 & 1.00 \\
\hline 0.11 & 1.11 \\
\hline- & 1.53 \\
\hline 2.47 & 1.00 \\
\hline 15.3 & 1.00 \\
\hline 1.01 & 0.996 \\
\hline 0.92 & 1.00 \\
\hline- & 1.73 \\
\hline 7.52 & 1.02 \\
\hline 0.02 & 1.10 \\
\hline 37.8 & 1.001 \\
\hline- & 1.25 \\
\hline 4.23 & 1.01 \\
\hline 1.07 & 1.03 \\
\hline
\end{tabular}

\begin{tabular}{|c|c|}
\hline $99 \% \mathrm{Cl}$ & $\boldsymbol{P}$ \\
\hline $1.07-1.11$ & $<0.001$ \\
\hline $0.92-1.80$ & 0.049 \\
\hline 0.97-1.06 & 0.364 \\
\hline $1.04-1.07$ & $<0.001$ \\
\hline $0.99-1.02$ & 0.020 \\
\hline $0.98-1.00$ & 0.085 \\
\hline- & - \\
\hline- & - \\
\hline- & - \\
\hline- & - \\
\hline- & - \\
\hline $1.35-1.72$ & $<0.001$ \\
\hline $1.02-1.18$ & 0.002 \\
\hline $0.68-1.31$ & 0.659 \\
\hline $1.09-2.13$ & 0.001 \\
\hline 0.99-1.01 & 0.044 \\
\hline $0.93-1.32$ & 0.126 \\
\hline $1.01-2.30$ & 0.008 \\
\hline $0.99-1.01$ & 0.316 \\
\hline $1.00-1.01$ & 0.002 \\
\hline $0.98-1.01$ & 0.366 \\
\hline $0.99-1.01$ & 0.685 \\
\hline $1.22-2.46$ & $<0.001$ \\
\hline $1.01-1.02$ & $<0.001$ \\
\hline $0.73-1.67$ & 0.541 \\
\hline $1.001-1.002$ & $<0.001$ \\
\hline $0.81-1.94$ & 0.187 \\
\hline 0.99-1.02 & 0.050 \\
\hline $0.99-1.07$ & 0.051 \\
\hline
\end{tabular}

$\mathrm{ACTH}$, adrenocorticotropic hormone; ARR, aldosterone-to-renin ratio; $\mathrm{BMI}$, body mass index; $\mathrm{Cl}$, confidence interval; eGFR, estimated glomerular filtration rate; HbA1c, Hemoglobin A1c; HDL, high-density lipoprotein; LDL, low-density lipoprotein; OR, odds ratio; PAC, plasma aldosterone concentration; PRA, plasma renin activity.

with PA and those with EHT; however, the prevalences of both overt and trace proteinuria were significantly higher among PA than EHT patients.

\section{Comparison between patients with the unilateral and bilateral subtype}

From among 2366 patients with PA who underwent AVS, 755 patients were excluded due to the following reasons: AVS performed without ACTH stimulation $(n=200)$; suspected autonomous cortisol secretion, which meant serum cortisol $\geq 1.8 \mu \mathrm{g} / \mathrm{dL}$ after a $1 \mathrm{mg}$ dexamethasone suppression test $\mathrm{mg} / \mathrm{dL}(n=351)$ and unsuccessful AVS, which meant a selectivity index $<5$ or no data on the selectivity index $(n=204)$. Consequently, 438 unilateral and 1173 bilateral patients were ultimately included in the study. We then compared the prevalences of renal impairment, proteinuria and lowered eGFR between patients with the unilateral or bilateral subtype (Supplementary Table 1, see section on supplementary data given at the end of this article). The prevalences of renal impairment and both overt and trace proteinuria were significantly higher in patients with the unilateral subtype than the bilateral subtype ( 26.4 vs $16.7 \%, 16.7$ vs $7.1 \%$, 36.2 vs $21.2 \%$, respectively). However, the prevalence of lowered eGFR did not statistically differ (13.1 vs 10.7\%).

\section{Discussion}

\section{Key results}

Using the largest database of PA patients in the world, we conducted a multi-institutional, retrospective, 
Table 6 Logistic regression analysis for renal impairment.

\begin{tabular}{l} 
Parameter \\
\hline Age, years \\
Sex, male \\
BMI, kg/m² \\
Duration of hypertension, years \\
Systolic blood pressure, mmHg \\
Na, mEq/L \\
Hypokalemia \\
Diabetes mellitus \\
Dyslipidemia \\
PAC, ng/dL
\end{tabular}

\begin{tabular}{c}
\hline Adjusted estimate \\
\hline 0.054 \\
-0.181 \\
0.064 \\
0.015 \\
0.010 \\
-0.008 \\
0.187 \\
0.138 \\
0.057 \\
0.021 \\
\hline
\end{tabular}

\begin{tabular}{c}
\hline Adjusted OR \\
\hline 1.06 \\
1.44 \\
1.07 \\
1.02 \\
1.01 \\
0.99 \\
1.45 \\
1.32 \\
1.12 \\
1.02
\end{tabular}

\begin{tabular}{c}
\hline $\mathbf{9 9} \% \mathbf{C l}$ \\
\hline $1.04-1.07$ \\
$1.03-1.99$ \\
$1.02-1.11$ \\
$0.99-1.03$ \\
$1.00-1.02$ \\
$0.92-1.07$ \\
$1.01-2.09$ \\
$0.89-1.96$ \\
$0.79-1.59$ \\
$1.01-1.03$
\end{tabular}

$\begin{array}{r}\hline \multicolumn{1}{c}{\boldsymbol{P}} \\ \hline<0.001 \\ 0.004 \\ <0.001 \\ 0.031 \\ 0.003 \\ 0.776 \\ 0.008 \\ 0.073 \\ 0.400 \\ <0.001 \\ \hline\end{array}$

$\mathrm{BMI}$, body mass index; $\mathrm{Cl}$, confidence interval; OR, odds ratio; PAC, plasma aldosterone concentration.

cross-sectional study to evaluate the contribution of PA to renal impairment, focusing on proteinuria and lowered eGFR.

Our study demonstrates that renal impairment in PA patients significantly correlates with the PAC level itself, which is unlike other cardiovascular complications. This finding suggests that aldosterone has a greater direct effect on the kidney than on other cardiovascular organs.

\section{Limitations}

There were several limitations to this study. First, this is a retrospective cross-sectional study. Because the prevalence of renal impairment would depend on the duration of the PA, to determine the exact incidences of renal impairment, proteinuria and lowered eGFR in patients with PA, we would need to perform a prospective study. However, it would be ethically difficult to prospectively follow patients with PA without administering specific treatment of PA. Second, the JPAS database is composed of data on PA patients and does not include data on EHT patients. To reduce the difference in the backgrounds between patients with PA and those with EHT, we used data from EHT patients who were treated as outpatients at the Kyoto Medical Center, which played a central role in the JPAS study. However, the clinical data for the EHT patients were not included in the JPAS study.

Because the EHT data were from a single center and were not registered in the same registry, we cannot exclude the possibility that they do not reflect the more generalized characteristics of patients with EHT. Third, proteinuria was examined in this study through qualitative, but not quantitative, urine analyses. Fourth, the investigators participating in the JPAS study were, in principle, expected to register all PA patients during the study period. However, those investigators were allowed to refrain from registering specific patients whom they judged to be inappropriate for our study. This could potentially cause a selection bias. Finally, renal impairment was diagnosed based on a one-time measurement. Consequently, we could not show whether the renal impairment was transient or persistent. We therefore refrained from using the term 'chronic kidney disease'. Instead we used 'renal impairment'.

Table 7 Logistic regression analysis for proteinuria.

\begin{tabular}{l} 
Parameter \\
\hline Age, year \\
Sex, male \\
BMI, kg/m² \\
Duration of hypertension, year \\
Systolic blood pressure, mmHg \\
Na, mEq/L \\
Hypokalemia \\
Diabetes mellitus \\
Dyslipidemia \\
PAC, ng/dL
\end{tabular}

\begin{tabular}{c}
\hline Adjusted estimate \\
\hline 0.014 \\
-0.357 \\
0.076 \\
0.023 \\
0.017 \\
-0.030 \\
0.423 \\
0.379 \\
0.062 \\
0.017
\end{tabular}

\begin{tabular}{c}
\hline Adjusted OR \\
\hline 1.01 \\
2.04 \\
1.08 \\
1.02 \\
1.02 \\
0.97 \\
2.33 \\
2.14 \\
1.13 \\
1.02 \\
\hline
\end{tabular}

\begin{tabular}{c}
\hline $\mathbf{9 9 \%} \mathbf{C l}$ \\
\hline $0.99-1.04$ \\
$1.32-3.16$ \\
$1.03-1.14$ \\
$0.99-1.05$ \\
$1.00-1.03$ \\
$0.88-1.07$ \\
$1.46-3.72$ \\
$1.31-3.47$ \\
$0.72-1.78$ \\
$1.01-1.03$ \\
\hline
\end{tabular}

$\begin{array}{r}\hline \multicolumn{1}{c}{\boldsymbol{P}} \\ \hline 0.120 \\ <0.001 \\ <0.001 \\ 0.015 \\ <0.001 \\ 0.416 \\ <0.001 \\ <0.001 \\ 0.483 \\ <0.001 \\ \hline\end{array}$

$\mathrm{BMI}$, body mass index; $\mathrm{Cl}$, confidence interval; OR, odds ratio; PAC, plasma aldosterone concentration.

https://eje.bioscientifica.com 
Table 8 Logistic regression analysis for lowered eGFR.

\begin{tabular}{l} 
Parameter \\
\hline Age, years \\
Duration of hypertension, years \\
$\mathrm{Na}, \mathrm{mEq} / \mathrm{L}$ \\
Hypokalemia \\
Diabetes mellitus \\
Dyslipidemia \\
PAC, ng/dL
\end{tabular}

\begin{tabular}{c}
\hline Adjusted estimate \\
\hline 0.080 \\
0.017 \\
-0.007 \\
0.043 \\
0.043 \\
0.059 \\
0.018 \\
\hline
\end{tabular}

\begin{tabular}{c}
\hline Adjusted OR \\
\hline 1.08 \\
1.02 \\
0.99 \\
1.09 \\
1.09 \\
1.13 \\
1.02 \\
\hline
\end{tabular}

\begin{tabular}{c}
\hline $\mathbf{9 9 \%} \mathbf{C l}$ \\
\hline $1.06-1.11$ \\
$0.99-1.04$ \\
$0.91-1.08$ \\
$0.71-1.68$ \\
$0.68-1.75$ \\
$0.75-1.69$ \\
$1.01-1.03$ \\
\hline
\end{tabular}

\begin{tabular}{r}
\multicolumn{1}{c}{$\boldsymbol{P}$} \\
\hline$<0.001$ \\
0.041 \\
0.857 \\
0.531 \\
0.624 \\
0.430 \\
$<0.001$
\end{tabular}

$\mathrm{Cl}$, confidence interval; eGFR, estimated glomerular filtration rate; OR, odds ratio; PAC, plasma aldosterone concentration.

\section{Interpretation}

Renal impairment associated with PA consists of two components (23). At an early stage of PA, increased sodium reabsorption and expansion of the extracellular fluid volume cause increased renal perfusion pressure and suppression of renin-angiotensin II. As a consequence, intrarenal vascular resistance decreases, leading to hyperfiltration. This is a functional adaptation, and structural renal damage is not obvious at this stage (24). The decrease in intrarenal vascular resistance at this stage was confirmed in a clinical study in which the echo Doppler technique was used to assess the resistance index for kidneys (25). The other component of renal impairment in PA reflects structural damage to the kidney resulting from persistent hypertension and hyperaldosteronism.

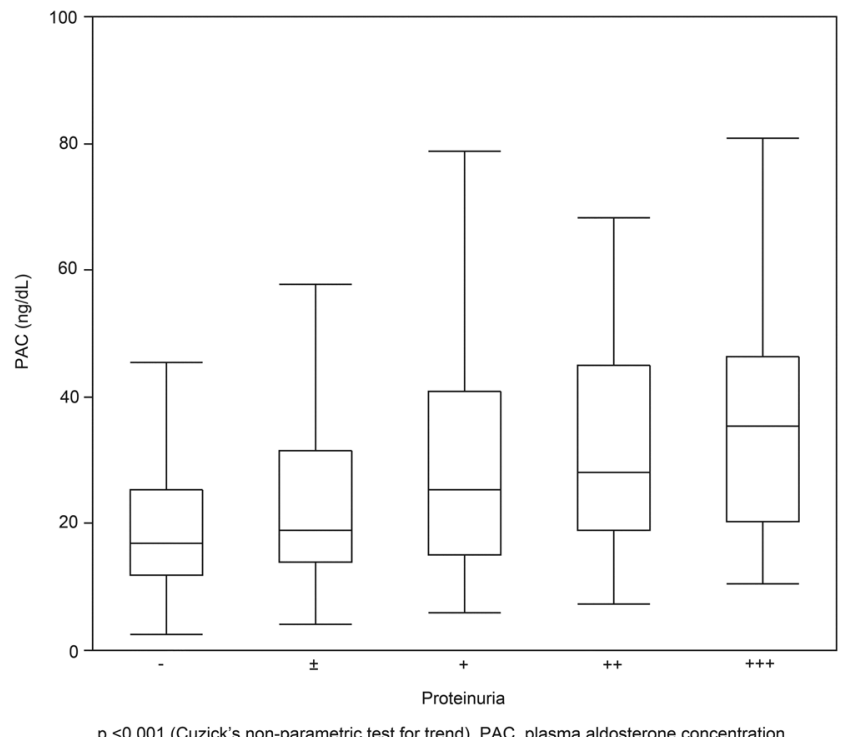

Figure 1

PACs in PA patients grouped according to their proteinuria level determined using a dip stick test. $P<0.001$ (Cuzick's non-parametric test for trend). PAC, plasma aldosterone concentration.
Hypertension causes fibrosis within intrarenal vessels (24), and hyperaldosteronism also causes adverse structural changes. Danforth et al. examined renal biopsy specimens from patients with PA and reported observing moderate renal tissue damage (26).

In the present study, the prevalence of renal impairment was $19.7 \%$. At first glance, this prevalence looks to be higher than that in the study from Iwakura et al., who reported the prevalence of CKD (chronic kidney disease) to be $11.7 \%$ in their cohort (27). However, their definition of renal impairment was only eGFR below $60 \mathrm{~mL} / \mathrm{min} / 1.73 \mathrm{~m}^{2}$; proteinuria was not included. In our study, the frequency of renal impairment was increased by inclusion of patients with overt proteinuria without lowered eGFR. In our study, the prevalence of eGFR below $60 \mathrm{~mL} / \mathrm{min} / 1.73 \mathrm{~m}^{2}$ was $11.6 \%$ or nearly the same as in the earlier study. The definition of renal insufficiency has also varied among other studies. In one case the criterion was a serum creatinine level $>1.5 \mathrm{mg} / \mathrm{dL}$ (28), while in another it was $1.25 \mathrm{mg} / \mathrm{dL}$ (9), and in a third it was based on creatinine clearance (CCr) (10). This makes it difficult to interpret the differences in the prevalence of renal insufficiency between the present study and earlier ones.

In the present study, the GFR in patients with PA did not differ from that in patients with EHT. Even when we calculated eGFR by using the CKD-EPI (the Chronic Kidney Disease Epidemiology Collaboration) formula (29), the difference was not observed (PA $78.2 \mathrm{~mL} / \mathrm{min} / 1.73 \mathrm{~m}^{2}$ vs EHT $79.9 \mathrm{~mL} / \mathrm{min} / 1.73 \mathrm{~m}^{2}$ ). It was observed in some earlier studies that the GFR is higher in patients with PA than with EHT $(10,11)$, while others report the GFR to be lower in PA than EHT patients $(9,7)$. And Ribstein et al. reported that the GFR does not differ between PA and EHT patients (12). This inconsistency may derive in part from differences in the severity of the renal impairment in each cohort. Steichen et al. reported that in patients with PA, the GFR declined after adrenalectomy or administration of a mineralocorticoid receptor antagonist (30). As discussed earlier, hyperfiltration due to hyperaldosteronism occurs 
Table 9 Comparison of patients with PA (JPAS Study) and EHT (Kyoto Medical Center) matched for age, sex, SBP and duration of hypertension. Data are presented as median (interquartile range).

\begin{tabular}{l}
\hline Characteristic \\
\hline Age, years \\
Sex, male, \% \\
BMI, kg/m² \\
Duration of hypertension, years \\
Systolic blood pressure, mmHg \\
Diastolic blood pressure, mmHg \\
Creatinine, mg/dL \\
Blood urea nitrogen, mg/dL \\
EGFR, mL/min/1.73 m² \\
Lowered eGFR, \% \\
Overt proteinuria, \% \\
Trace and overt proteinuria, \% \\
Renal impairment, \% \\
Uric acid, mg/dL \\
Na, mEq/L \\
K, mEq/L \\
Diabetes mellitus, \% \\
Dyslipidemia, \% \\
PAC, ng/dL \\
PRA, ng/mL/h \\
ARR, ng/dL per ng/mL/h
\end{tabular}

\begin{tabular}{c}
\hline Patients with PA \\
\hline$n=128$ \\
\hline 59 (47-66) \\
$49.2 \%$ \\
$24.1(21.5-26.3)$ \\
$3(1-9)$ \\
$140(129-152)$ \\
$87(80-92)$ \\
$0.71(0.60-0.84)$ \\
$13.5(11.0-16.2)$ \\
$76.1(65.1-87.8)$ \\
$17.2 \%$ \\
$16.8 \%$ \\
$31.1 \%$ \\
$28.9 \%$ \\
$5.3(4.3-6.1)$ \\
$142(141-143)$ \\
$3.9(3.4-4.1)$ \\
$18.8 \%$ \\
$28.1 \%$ \\
$15.7(11.8-25.2)$ \\
$0.3(0.2-0.6)$ \\
$46(30-91)$ \\
\end{tabular}

\begin{tabular}{ccr}
\hline Patients with EHT & & $\boldsymbol{P}$ \\
\hline$n=128$ & & 0.999 \\
$(47-66)$ & & 1.000 \\
$49.2 \%$ & 0.394 \\
$23.2(21.4-26.7)$ & 0.898 \\
$3(1-9)$ & & 0.950 \\
$140(130-151)$ & 0.995 \\
$86(79-92)$ & & 0.940 \\
$0.70(0.60-0.90)$ & 0.175 \\
$14.0(12.0-17.0)$ & 0.970 \\
$75.0(65.4-86.7)$ & 0.628 \\
$15.0 \%$ & 0.002 \\
$4.4 \%$ & 0.002 \\
$13.9 \%$ & 0.079 \\
$19.1 \%$ & 0.322 \\
$5.5(4.4-6.5)$ & $<0.001$ \\
$140(139-141)$ & $<0.001$ \\
$4.1(3.9-4.3)$ & 0.904 \\
$19.6 \%$ & 0.415 \\
$32.8 \%$ & 0.041 \\
$14.9(11.2-18.0)$ & $<0.001$ \\
$2.4(1.4-4.6)$ & $<0.001$ \\
$6.5(3.6-10.3)$ &
\end{tabular}

ARR, aldosterone-to-renin ratio; BMI, body mass index; eGFR, estimated glomerular filtration rate; PAC, plasma aldosterone concentration; PRA, plasma renin activity.

in patients with PA. We therefore suggest the GFR is actually lower in patients with PA than with EHT, but hyperfiltration masks the true magnitude of the decrease.

There have been several studies focusing on the pathogenic role of aldosterone in renal injury. Blasi et al. showed that aldosterone infusion and salt intake cause hypertension, intrarenal vascular and glomerular sclerosis, fibrinoid necrosis, tubular damage and albuminuria (8). Similar results have been reported by others $(5,7$, 31). Nagase et al. reported that podocytes, which act against urinary protein loss, are damaged by a high-salt diet with aldosterone infusion in uninephrectomized rats. As a result, these rats exhibit massive proteinuria (32). In the present study, the prevalences of overt and trace proteinuria were 10.3 and $25.7 \%$, respectively. We also found that the prevalence of proteinuria, whether overt or trace, was significantly higher in PA than EHT patients, which is consistent with earlier results $(10,11$, 12). Catena et al. reported that this difference was due to hyperfiltration caused by hyperaldosteronism, as the difference was normalized after adjusting for the urinary creatinine concentration (10). In the present study, however, the prevalences of overt and trace proteinuria were significantly higher in patients with PA, though eGFR in those patients did not differ from that in patients with EHT. This result suggests that the difference in the prevalence of proteinuria in our cohort is not explained by hyperfiltration. We suggest that eGFR is actually lower in patients with PA than with EHT, but hyperfiltration masks the true decline and that proteinuria is a hallmark of renal impairment in patients with PA. In our analysis, when patients were divided into five groups according to their level of proteinuria, we found that as the PAC was significantly increased, so was the level of proteinuria (Fig. 1).

This suggests a positive relationship between the PAC and the severity of proteinuria. We recently reported that the PAC is not itself associated with cardiovascular events such as stroke or ischemic heart disease (4). To the contrary, however, analyzing the same JPAS patients in the present study, the PAC was significantly associated with the odds of renal impairment, proteinuria and lowered eGFR. Although aldosterone influences both the cardiovascular system and kidney, we suggest that the mechanism by which aldosterone affects the kidney differs from the mechanism affecting the vasculature itself and that the PAC may have a more direct effect on the kidney than on other cardiovascular organs.

\section{Supplementary data}

This is linked to the online version of the paper at https://doi.org/10.1530/ EJE-19-0047. 


\section{Declaration of interest}

The authors declare that there is no conflict of interest that could be perceived as prejudicing the impartiality of this study.

\section{Funding}

This research was supported by a research grant from the Japan Agency for Medical Research and Development (AMED) under grant numbers JP17ek0109122 and JP18ek0109352. This research was also supported by a grant from the Ministry of Health, Labour and Welfare, Japan (Jitsuyoka Nanbyo-Ippan-046).

\section{Acknowledgements}

The acknowledgement list of JPAS and JRAS groups is available in the supplementary data of this article.

\section{References}

1 Funder JW, Carey RM, Mantero F, Murad MH, Reincke M, Shibata H, Stowasser M \& Young Jr WF. The management of primary aldosteronism: case detection, diagnosis, and treatment: an Endocrine Society practice guideline. Journal of Clinical Endocrinology and Metabolism 2016101 1889-1916. (https://doi.org/10.1210/ jc.2015-4061)

2 Milliez P, Girerd X, Plouin PF, Blacher J, Safar ME \& Mourad JJ. Evidence for an increased rate of cardiovascular events in patients with primary aldosteronism. Journal of the American College of Cardiology 200545 1243-1248. (https://doi.org/10.1016/j. jacc.2005.01.015)

3 Mulatero P, Monticone S, Bertello C, Viola A, Tizzani D, Iannaccone A, Crudo V, Burrello J, Milan A, Rabbia F et al. Longterm cardio- and cerebrovascular events in patients with primary aldosteronism. Journal of Clinical Endocrinology and Metabolism 2013 98 4826-4833. (https://doi.org/10.1210/jc.2013-2805)

4 Ohno Y, Sone M, Inagaki N, Yamasaki T, Ogawa O, Takeda Y, Kurihara I, Itoh $\mathrm{H}$, Umakoshi $\mathrm{H}$, Tsuiki M et al. Prevalence of cardiovascular disease and its risk factors in primary aldosteronism: a multicenter study in Japan. Hypertension 201871 530-537. (https:// doi.org/10.1161/HYPERTENSIONAHA.117.10263)

5 Nishiyama A, Yao L, Nagai Y, Miyata K, Yoshizumi M, Kagami S, Kondo S, Kiyomoto H, Shokoji T, Kimura S et al. Possible contributions of reactive oxygen species and mitogen-activated protein kinase to renal injury in aldosterone/salt-induced hypertensive rats. Hypertension 200443 841-848. (https://doi. org/10.1161/01.HYP.0000118519.66430.22)

6 Rocha R, Chander PN, Khanna K, Zuckerman A \& Stier Jr CT. Mineralocorticoid blockade reduces vascular injury in stroke-prone hypertensive rats. Hypertension 199831 451-458. (https://doi. org/10.1161/01.HYP.31.1.451)

7 Rocha R, Chander PN, Zuckerman A \& Stier Jr CT. Role of aldosterone in renal vascular injury in stroke-prone hypertensive rats. Hypertension 199933 232-237. (https://doi.org/10.1161/01. HYP.33.1.232)

8 Blasi ER, Rocha R, Rudolph AE, Blomme EA, Polly ML \& McMahon EG. Aldosterone/salt induces renal inflammation and fibrosis in hypertensive rats. Kidney International 200363 1791-1800. (https://doi.org/10.1046/j.1523-1755.2003.00929.x)

9 Reincke M, Rump LC, Quinkler M, Hahner S, Diederich S, Lorenz R, Seufert J, Schirpenbach C, Beuschlein F, Bidlingmaier M et al. Risk factors associated with a low glomerular filtration rate in primary aldosteronism. Journal of Clinical Endocrinology and Metabolism 2009 94 869-875. (https://doi.org/10.1210/jc.2008-1851)
10 Catena C, Colussi G, Nadalini E, Chiuch A, Baroselli S, Lapenna R \& Sechi LA. Relationships of plasma renin levels with renal function in patients with primary aldosteronism. Clinical Journal of the American Society of Nephrology 20072 722-731. (https://doi.org/10.2215/ CJN.00050107)

11 Sechi LA, Novello M, Lapenna R, Baroselli S, Nadalini E, Colussi GL \& Cateca C. Long-term renal outcomes in patients with primary aldosteronism. JAMA 2006295 2638-2645. (https://doi.org/10.1001/ jama.295.22.2638)

12 Ribstein J, Du Cailar G, Fesler P \& Mimran A. Relative glomerular hyperfiltration in primary alsoteronism. Journal of the American Society of Nephrology 200516 1320-1325. (https://doi.org/10.1681/ ASN.2004100878)

13 Rossi GP, Bernini G, Caliumi C, Desideri G, Fabris B, Ferri C, Ganzaroli C, Giacchetti G, Letizia C, Maccario M et al. A prospective study of the prevalence of primary aldosteronism in 1,125 hypertensive patients. Journal of the American College of Cardiology 200648 2293-2300. (https://doi.org/10.1016/j.jacc.2006.07.059)

14 Chronic Kidney Disease Prognosis Consortium, Matsushita K, van der Velde M, Astor BC, Woodward M, Levey AS, de Jong PE, Coresh J \& Gansevoort RT. Association of estimated glomerular filtration rate and albuminuria with all-cause and cardiovascular mortality in general population cohorts: a collaborative meta-analysis. Lancet 2010375 2073-2081. (https://doi.org/10.1016/S01406736(10)60674-5)

15 Japan Nephrology Society. Special issue: Clinical practice guidebook for diagnosis and treatment of chronic kidney disease 2012 (in Japanese). Nihon Jinzo Gakkai Shi 201254 1034-1191.

16 Nishikawa T, Omura M, Satoh F, Shibata H, Takahashi K, Tamura N, Tanabe A, Task Force Committee on Primary Aldosteronism \& The Japan Endocrine Society. Guidelines for the diagnosis and treatment of primary aldosteronism - the Japan Endocrine Society 2009. Endocrine Journal 201158 711-721. (https://doi.org/10.1507/endocrj. EJ11-0133)

17 Shimamoto K, Ando K, Fujita T, Hasebe N, Higaki J, Horiuchi M, Imai Y, Imaizumi T, Ishimitsu T, Ito $\mathrm{M}$ et al. The Japanese Society of Hypertension guideline for the management of hypertension (JSH 2014). Hypertension Research 201437 253-390. (https://doi. org/10.1038/hr.2014.20)

18 Young WF, Stanson AW, Thompson GB, Grant CS, Farley DR \& van Heerden JA. Role for adrenal venous sampling in primary aldosteronism. Surgery 2004136 1227-1235. (https://doi. org/10.1016/j.surg.2004.06.051)

19 Yanase T, Oki Y, Katabami T, Otsuki M, Kageyama K, Tanaka T, Kawate H, Tanabe M, Doi M, Akehi Y et al. New diagnostic criteria of adrenal subclinical Cushing's syndrome: opinion from the Japan Endocrine Society. Endocrine Journal 201865 383-393. (https://doi. org/10.1507/endocrj.EJ17-0456)

20 Seino Y, Nanjo K, Tajima N, Kadowaki T, Kashiwagi A, Araki E, Ito C, Inagaki $\mathrm{N}$, Iwamoto $\mathrm{Y}$, Kasuga $\mathrm{M}$ et al. Report of the committee on the classification and diagnosis of diabetes mellitus. Journal of Diabetes Investigation 20101 212-228. (https://doi.org/10.1111/ j.2040-1124.2010.00074.x)

21 Teramoto T, Sasaki J, Ishibashi S, Birou S, Daida H, Dohi S, Egusa G, Hiro T, Hirobe K, Iida $\mathrm{M}$ et al. Diagnostic criteria for dyslipidemia. Journal of Atherosclerosis and Thrombosis 201320 655-660. (https:// doi.org/10.5551/jat.17152)

22 Pocock SJ, MuMurray JJ \& Collier TJ. Making sense of statistics in clinical trial reports: part 1 of a 4-part series on statistics for clinical trials. Journal of the American College of Cardiology 201566 2536-2549. (https://doi.org/10.1016/j.jacc.2015.10.014)

23 Catena C, Colussi G \& Sechi LA. Aldosterone, organ damage and dietary salt. Clinical and Experimental Pharmacology and Physiology 201340 922-928. (https://doi.org/10.1111/1440-1681.12145)

24 Sechi LA, Colussi G, Di Fabio A \& Cateba C. Cardiovascular and renal damage in primary aldosteronism: outcomes after treatment. 
American Journal of Hypertension 201023 1253-1260. (https://doi org/10.1038/ajh.2010.169)

25 Sechi LA, Di Fabio A, Bazzocchi M, Uzzau A \& Catena C. Intrarenal hemodynamics in primary aldosteronism before and after treatment. Journal of Clinical Endocrinology and Metabolism 200994 1191-1197. (https://doi.org/10.1210/jc.2008-2245)

26 Danforth Jr DN, Orlando MM, Bartter FC \& Javadpour N. Renal changes in primary aldosteronism. Journal of Urology $1977 \mathbf{1 1 7}$ 140-144. (https://doi.org/10.1016/s0022-5347(17)58376-7)

27 Iwakura Y, Morimoto R, Kudo M, Ono Y, Takase K, Seiji K, Arai Y, Nakamura Y, Sasano H, Ito $\mathrm{S}$ et al. Predictors of decreasing glomerular filtration rate and prevalence of chronic kidney disease after treatment of primary aldosteronism: renal outcome of 213 cases. Journal of Clinical Endocrinology and Metabolism 201499 1593-1598. (https://doi.org/10.1210/jc.2013-2180)

28 Nishimura M, Uzu T, Fujii T, Kuroda S, Nakamura S, Inenaga T \& Kimura G. Cardiovascular complications in patients with primary aldosteronism. American Journal of Kidney Diseases 199933 261-266. (https://doi.org/10.1016/S0272-6386(99)70298-2)

29 Horio M, Imai E, Yasuda Y, Watanabe T \& Matsuo S. Modification of the CKD Epidemiology Collaboration (CKD-EPI) equation for Japanese: accuracy and use for population estimates. American Journal of Kidney Diseases 201056 32-38. (https://doi.org/10.1053/j.ajkd.2010.02.344)

30 Steichen O, Lorthioir A, Zinzindohoue F, Plouin PF \& Amar L. Outcomes of drug-based and surgical treatments for primary aldosteronism. Advances in Chronic Kidney Disease 201522 196-203. (https://doi.org/10.1053/j.ackd.2014.10.003)

31 Liang W, Chen C, Shi J, Ren Z, Hu F, van Goor H, Singhal PC \& Ding G. Disparate effects of eplerenone, amlodipine and telmisartan on podocyte injury in aldosterone-infused rats. Nephrology, Dialysis, Transplantation 201126 789-799. (https://doi.org/10.1093/ndt/gfq514)

32 Nagase M \& Fujita T. Aldosterone and glomerular podocyte injury. Clinical and Experimental Nephrology 200812 233-242. (https://doi. org/10.1007/s10157-008-0034-9)

Received 26 January 2019

Revised version received 10 July 2019

Accepted 10 July 2019 